\title{
An Updated Catalog of 4680 Northern Eclipsing Binaries with Algol-type Light-curve Morphology in the Catalina Sky Surveys
}

\author{
Athanasios Papageorgiou ${ }^{1,2}$ (10, Márcio Catelan ${ }^{1,2,5}$ (10), Panagiota-Eleftheria Christopoulou ${ }^{3}$, Andrew J. Drake ${ }^{4}$, and \\ S. G. Djorgovski ${ }^{4}$ (1) \\ ${ }^{1}$ Pontificia Universidad Católica de Chile, Facultad de Física, Instituto de Astrofísica, Av. Vicuña Mackenna 4860, 782-0436 Macul, Santiago, Chile \\ apapageo@astro.puc.cl \\ ${ }^{2}$ Millennium Institute of Astrophysics, Santiago, Chile \\ ${ }^{3}$ Department of Physics, University of Patras, 26500, Patra, Greece \\ ${ }^{4}$ California Institute of Technology, 1200 East California, Boulevard, CA 91225, USA \\ Received 2018 April 14; revised 2018 June 30; accepted 2018 July 21; published 2018 September 5
}

\begin{abstract}
We present an updated catalog of 4680 northern eclipsing binaries (EBs) with Algol-type light-curve (LC) morphology (i.e., with well-defined beginnings and ends of primary and secondary eclipses), using data from the Catalina Sky Surveys. Our work includes revised period determinations, phenomenological parameters of the LCs, and system morphology classifications based on machine-learning techniques. While most of the new periods are in excellent agreement with those provided in the original Catalina catalogs, improved values are now available for $\sim 10 \%$ of the stars. A total of 3456 EBs were classified as detached and 449 were classified as semi-detached, while 145 could not be classified unambiguously into either subtype. The majority of the SD systems seem to be comprised of short-period Algols. By applying color criteria, we searched for K- and M-type dwarfs in these data, and present a subsample of $609 \mathrm{~EB}$ candidates for further investigation. We report 119 EBs $(2.5 \%$ of the total sample) that show maximum quadrature light variations over long timescales, with periods bracketing the range $4.5-18$ years and a fractional luminosity variance range of $0.04-0.13$. We discuss possible causes for this, making use of models of variable starspot activity in our interpretation of the results.
\end{abstract}

Key words: binaries: eclipsing - catalogs - methods: data analysis - surveys

Supporting material: machine-readable tables

\section{Introduction}

Our thinking about eclipsing binary stars (EBs) has undergone a tremendous change in the last decade. EBs are one of nature's best laboratories for determining the fundamental physical properties of stars, and thus for testing the predictions of theoretical models (e.g., Torres et al. 2010; Catelan \& Smith 2015, and references therein). A large number of eclipsing Algol-type (EA) binaries, for which the beginnings and ends of eclipses are well defined, have been discovered recently as a by-product of several wide-field, ground-based photometric surveys, some of which are dedicated to the detection of variable stars. Among these surveys, one finds the Catalina Sky Survey (CSS, Larson et al. 2003), the Visible and Infrared Survey Telescope for Astronomy (VISTA) Variables in the Via Lactea (VVV, Minniti et al. 2010; Catelan et al. 2013), the asteroid survey LINEAR (Stokes et al. 2000; Palaversa et al. 2013), the All Sky Automated Survey (ASAS, Pojmanski 1997; Pojmanski et al. 2005), the Northern Sky Variability Survey (NSVS, Woźniak et al. 2004), the Transatlantic Exoplanet Survey (TrES, Alonso et al. 2004, 2007), the Optical Gravitational Lensing Experiment (OGLE, Udalski et al. 1992) survey, the Hungarian-made Automated Telescope Network exoplanet survey (HATNet, Bakos et al. 2004), and the Wide Angle Search for Planets (SuperWASP, Christian et al. 2006; Pollacco et al. 2006), among others (see Kovacs 2017; Soszyński 2017, for recent reviews and references).

\footnotetext{
${ }^{5}$ On sabbatical leave at The Observatories of the Carnegie Institution for Science, 813 Santa Barbara Street, Pasadena, CA 91101, USA.
}

On the basis of light-curve (LC) morphology, EA-type eclipsing systems, with clearly defined eclipses on their LCs, include both (D) detached and semi-detached (SD) systems. As a rule, in order to establish the actual system configuration of any individual EB with such an Algol-type LC morphology, a detailed physical modeling is required.

The aforementioned projects are very useful for understanding the photometric properties of the different types of binaries, affording, for instance, statistical studies of the properties of EA systems. In addition, large samples provide the opportunity for special cases of binaries that need dedicated follow-up observations to emerge, or even to reveal new classes (e.g., the Heartbeat stars, Welsh et al. 2011).

In this work, we use the northern data from CSS (which continues collecting data to this day) in order to complete our search for detached EBs and to present an updated and more detailed catalog of their properties, in comparison with Drake et al. (2009, 2014a, 2014b, 2017). The additional data allow us not only to revise their periods and class but also to derive the phenomenological and physical parameters of selected detached systems. Furthermore, we were able to search for systems exhibiting long-term variation, which also might potentially harbor low-mass components.

This paper, the first in a series on the subject, is organized as follows. In Section 2, we briefly describe the CSS data that we use in our analysis. The construction of the sample and an outline of the analysis methods are explained in Section 3. Estimates of the periods and morphological features, and a physical classification of the EA type, are given in Section 4. In Sections 5 and 6 , we discuss all the results, followed by a brief summary of our work in Section 7. 


\section{Observations}

Observations were carried out during 2004-2016 using the three telescopes of the CSSs of Drake et al. (2009), ${ }^{6}$ covering the sky declination range $\delta=[-75,+65] \mathrm{deg}$, but avoiding crowded stellar regions within $10^{\circ}-15^{\circ}$ of the Galactic plane. The main goal of the survey is to discover near-Earth objects and potential hazardous asteroids. Nevertheless, time-series photometry for $\sim 200$ million variable sources has been accumulated through CSS. In order to maximize the throughput, the observations are taken unfiltered, and the magnitudes are transformed to an approximate $V$ magnitude $\left(V_{\mathrm{CSs}}\right.$; Drake et al. 2013). The photometry was performed using the aperture photometry program SExtractor (Bertin \& Arnouts 1996).

In this study, we use Catalina Surveys Data Release $2^{7}$ (CSDR2) with additional, not publicly accessible data, spanning 12 years (2004-2016). We focus on the sample of Drake et al. (2014b) of 4683 eclipsing binaries originally classified as EA type on the basis of 8 years of data. These cover a region of right ascension (R.A.) between 0 and $24 \mathrm{hr}$ and declination (decl.) between $-22^{\circ}$ and $+65^{\circ}$, as shown in Figure 1 (top). The bottom panel of Figure 1 shows the distribution of the new available data used in the present paper against the CSDR2 data. The total number of photometric points for the candidate systems that we studied significantly exceeds that available in the previous release.

\section{Identification of Algol-type Eclipsing Binaries in Catalina Sky Survey}

As we wanted to take all good data points of an LC into account to search for periodic signals, we first cleaned the 4683 LCs of the initial sample. For every LC a sigma-clipping cutoff algorithm was used to discard erroneous data points with values outside the interval of $\pm 5 \sigma$ of the median relative flux, where $\sigma$ denotes the standard deviation computed from the whole LC. Furthermore, by adopting a pre-defined period from Drake et al. (2014b), we performed $5 \sigma$ clipping from the median value of each phase bin. Therefore, we avoid rejecting data points corresponding to an eclipse and we ensure that the data points with errors larger than $5 \sigma$ are discarded as outliers, presumably due to unreliable measurements.

\subsection{Period Search}

After cleaning and checking a certain number of the resultant LCs, we applied a series of period-finding methods:

1. Analysis of Variance (AoV, Schwarzenberg-Czerny 1989, and Devor 2005);

2. Box-Least Squares (BLS, Kovács et al. 2002);

3. Generalized Lomb-Scargle (GLS, Press et al. 1992; Zechmeister \& Kürster 2009);

4. Phase Dispersion Minimization (PDM, Stellingwerf 1978); and

5. Correntropy Kernelized Periodogram (CKP, Protopapas et al. 2015).

The AoV, BLS, and GLS algorithms were applied through the command line utility VARTOOLS (Hartman \& Bakos 2016).

At first, the AoV method was applied, using a period range of [0.1-700] days and frequency resolution $0.1 / T$ (where $T$ is

\footnotetext{
6 http://catalinadata.org

7 http://nesssi.cacr.caltech.edu/DataRelease/
}

the time span of the LC), returning the top 5 peaks of the spectrum. The phase-folded LCs were visual inspected using these periods, and the best values were adopted. When the period values from AoV failed to phase-fold the LCs adequately, the other methods were applied, and the phased LCs were again visually inspected. A common issue encountered using periodograms, in the case of EBs, is the double/half period detection. For this reason, the majority of the phasefolded LCs were also examined using twice/half the detected period.

Using the methodology described, we improved the period determination of the detached EB sample. While most of the new periods are in excellent agreement with those provided in Drake et al. (2014b), improved values are now available for $\sim 10 \%$ of the 4680 stars (Figure 2). Table 1 summarizes the results obtained for the latter, also including also the mean photometric error $\left(V_{\text {err }}\right)^{8}$ and source coordinates (R.A.J2000, decl.J2000).

\subsection{LC Phenomenological Parameters}

After phase-folding the LCs as explained in the previous subsection, long-term variations were also removed, when present (see Section 5). The LC was then fitted using the LMFIT $^{9}$ (Newville et al. 2016) module in Python ${ }^{10}$ in order to derive its morphological features. Three different models were used, namely a chain of second-order polynomials (Prša et al. 2008; Papageorgiou et al. 2014), Fourier series fitting and a two-Gaussian model (TGM, Mowlavi et al. 2017). The actual fitting process was overseen by the Levenberg-Marquardt (LM, Levenberg 1944; Marquardt 1963) nonlinear minimization algorithm. We found that the TGM technique was much more robust and efficient, when applied to the stars in our sample. The procedure is based on modeling the geometry of LCs using Gaussian functions (to model the eclipses) and a cosine function (to model ellipsoidal variability, if present). Fitting a TGM to a time-series is very sensitive to the adopted initial values of the parameters. We therefore used the LM parameter values as starting points on each TGM. These included the phases $\left(\mu_{i}\right)$, the half widths $\left(s_{i}\right)$, and the depths $\left(d_{i}\right)$ of the primary and secondary eclipses $(i=1,2)$, the peak-to-peak amplitude of the ellipsoidallike variation $\left(A_{\mathrm{ell}}\right)$, and a constant $(C)$ that equals the maximum light of the LC in the case of detached systems (Mowlavi et al. 2017, see their Figure 1).

Since the success of modeling the folded LCs depends on the time sampling, measurement uncertainties, initial guessing of eclipse locations, and additional intrinsic variability in one or both stars of the binary system, a Markov Chain Monte Carlo (MCMC) analysis was performed on each TGM of our LC

\footnotetext{
8 The original CSS photometric errors are significantly overestimated, as discussed in Graham et al. (2017). Graham et al. provide a corrective factor $f_{\text {cor }}$ to compensate for this problem. The following analytical fit provides an excellent description of the data shown in Figure 1 of their paper:

$$
f_{\text {corr }}=a\left[1+\frac{(V-b)^{2}}{c^{2} d}\right]^{-\left(\frac{d}{2}+\frac{1}{2}\right)},
$$

with $a=1.350, b=19.491, c=3.006$, and $d=0.275$. The fit is valid between $V=14.0$ and $19.5 \mathrm{mag}$. For $V<14.0 \mathrm{mag}$, a value $f_{\text {corr }}=0.26$ is assumed; for $V>19.50 \mathrm{mag}$, we adopt instead $f_{\text {corr }}=1.35$. All error values reported in this paper, including tables and plots, have been corrected according to this recipe.

9 https://doi.org/10.5281/zenodo. 11813

10 http://www.python.org
} 

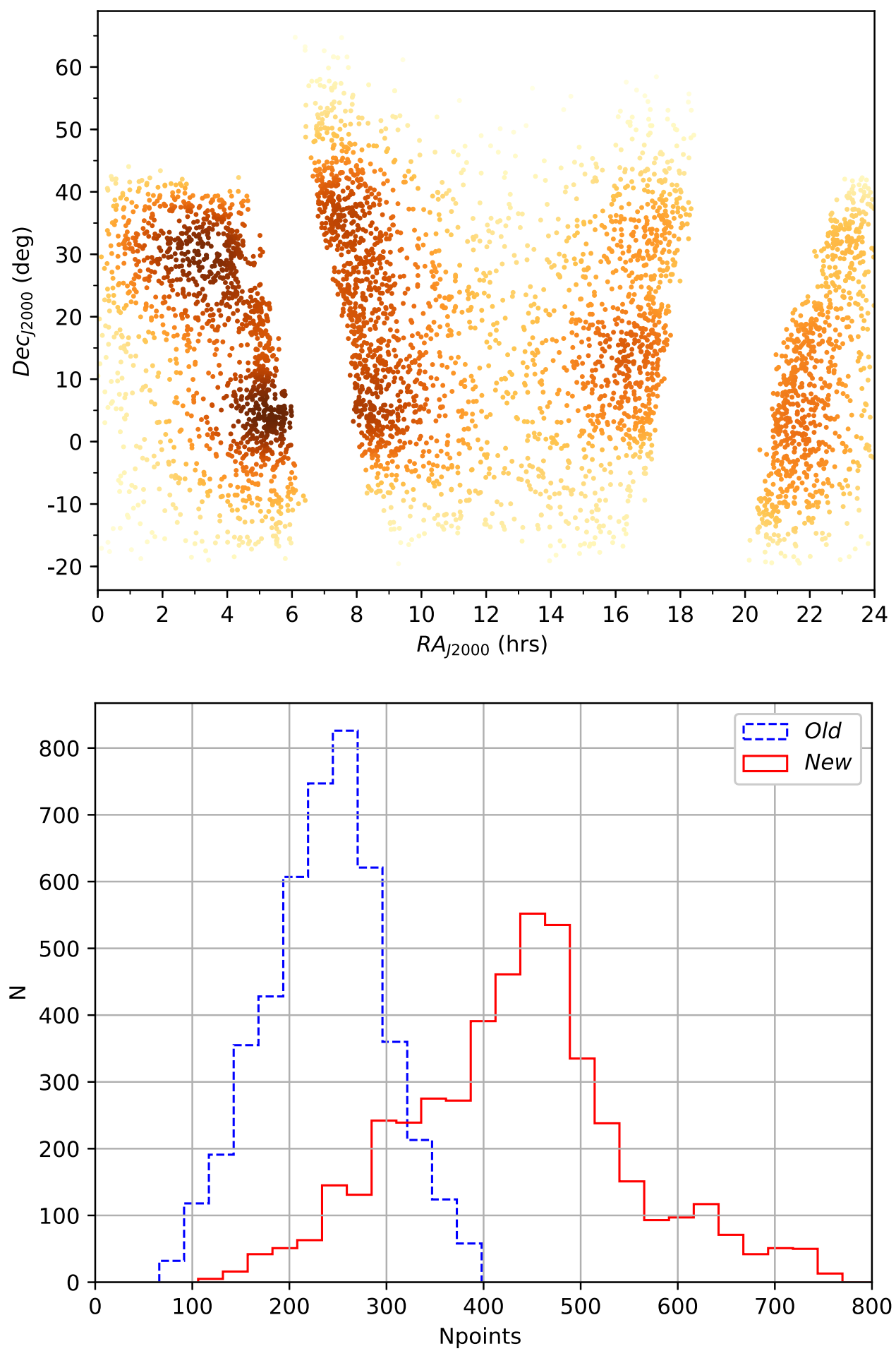

Figure 1. Top: sky distribution of $4683 \mathrm{EBs}$ in the CSS catalog. Bottom: distribution of the total number of photometric points per LC. The binary systems from the previous (Drake et al. 2014b) and new data releases (with the additional available data) are marked in the blue and red histograms, respectively.

sample, using the pyMC (Fonnesbeck et al. 2015) module ${ }^{11}$ in Python. The MCMC process begins by generating initial guesses for all the parameters randomly selected from a normal distribution based on the final LM fitting parameter values and errors. The new fit is accepted or rejected using the MetropolisHastings algorithm (Hastings 1970), compared to the fitting carried out in the previous step. In order to avoid the biases that

\footnotetext{
11 https://pymc-devs.github.io/pymc/
}

might be present in the initial solutions, the first 15,000 steps (of 200,000 steps in total) were discarded in the process. We then sampled this new synthetic model and discovered that the initial TGM model was noticeably different in some cases (Figure 3). Examples of the folded LCs, classified as D and SD as discussed below, are presented in Figure 4. The derived phenomenological parameters for $4680 \mathrm{EBs}$ are presented in Table 2. Such parameters include: the magnitude at primary (MinI) and secondary eclipse (MinII), the magnitude at 


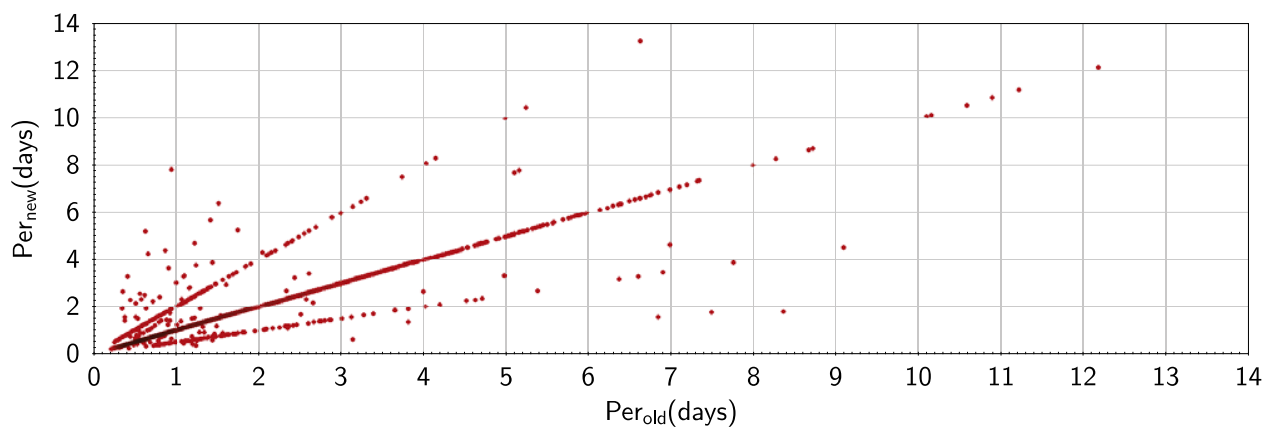

Figure 2. Refined periods of 4680 EB stars. While most of the new periods $\left(\right.$ Per $\left._{\text {new }}\right)$ are in excellent agreement with those provided in Drake et al. (2014a, Per ${ }_{\text {old }}$ ), improved values are now available for $\sim 10 \%$ of the stars. The differences are mostly due to aliases.

Table 1

CRTS EA Systems

\begin{tabular}{|c|c|c|c|c|c|c|c|c|c|c|}
\hline Name & ID & $\begin{array}{l}\text { R.A. } \\
\text { (h:m:s) }\end{array}$ & $\begin{array}{c}\text { Decl. } \\
\left({ }^{\circ}:{ }^{\prime}:{ }^{\prime \prime}\right)\end{array}$ & $\begin{array}{l}\text { MJD }^{\mathrm{a}} \\
\text { (days) }\end{array}$ & $\begin{array}{l}\text { Per } \\
\text { (days) }\end{array}$ & $\begin{array}{l}\left\langle V_{\text {err }}\right)^{\mathrm{b}} \\
(\mathrm{mag})\end{array}$ & Npoints & Class & LMCand & LongTerm \\
\hline CSS_J235945.5+303731 & 11291130655 & $23: 59: 45.5$ & $+30: 37: 31.8$ & 54265.53875 & 2.68651 & 0.0138 & 391 & $\mathrm{D}$ & $\ldots$ & $\cdots$ \\
\hline CSS_J235856.7+371823 & 11381030196 & $23: 58: 56.7$ & $+37: 18: 23.5$ & 55062.35518 & 1.35464 & 0.0136 & 312 & $\mathrm{D}$ & $\cdots$ & $\cdots$ \\
\hline CSS_J235816.7+293325 & 11291130352 & $23: 58: 16.7$ & $+29: 33: 25.3$ & 53537.41966 & 0.72949 & 0.0457 & 390 & SD & $\cdots$ & $\cdots$ \\
\hline CSS_J235756.9-023247 & 10011280051 & $23: 57: 56.9$ & $-02: 32: 47.2$ & 54747.25303 & 1.74457 & 0.0133 & 325 & $\mathrm{~N} / \mathrm{A}$ & $\cdots$ & $\cdots$ \\
\hline CSS_J235715.5+305455 & 11291130739 & $23: 57: 15.5$ & $+30: 54: 55.4$ & 53563.82135 & 2.84991 & 0.0157 & 381 & $\mathrm{D}$ & $\cdots$ & $\cdots$ \\
\hline CSS_J235538.3+384723 & 11381030654 & $23: 55: 38.3$ & $+38: 47: 23.1$ & 55508.59468 & 0.46792 & 0.0356 & 312 & $\mathrm{~N} / \mathrm{A}$ & $\ldots$ & $\ldots$ \\
\hline CSS_J235444.8+305751 & 11291130763 & $23: 54: 44.8$ & $+30: 57: 51.9$ & 54394.71148 & 0.82131 & 0.0279 & 349 & $\mathrm{D}$ & $\cdots$ & $\ldots$ \\
\hline CSS_J235401.4+374029 & 11381030304 & 23:54:01.4 & $+37: 40: 29.7$ & 56558.31886 & 0.50473 & 0.0232 & 312 & $\mathrm{D}$ & $\cdots$ & $\cdots$ \\
\hline CSS_J235313.6-021850 & 10011280091 & $23: 53: 13.6$ & $-02: 18: 50.2$ & 53655.26418 & 0.50952 & 0.0152 & 325 & $\mathrm{D}$ & $\cdots$ & $\cdots$ \\
\hline CSS_J235227.0+395515 & 11400990133 & $23: 52: 27.0$ & $+39: 55: 15.3$ & 53694.94006 & 1.5311 & 0.0287 & 218 & $\mathrm{D}$ & $\ldots$ & $\ldots$ \\
\hline CSS_J235151.3+035409 & 11041280214 & $23: 51: 51.3$ & $+03: 54: 09.0$ & 55850.15160 & 2.98858 & 0.0136 & 375 & $\mathrm{D}$ & $\cdots$ & $\cdots$ \\
\hline CSS_J235104.0+115651 & 11121260136 & 23:51:04.0 & $+11: 56: 51.3$ & 54095.14156 & 0.81240 & 0.0138 & 442 & D & $\cdots$ & $\cdots$ \\
\hline CSS_J234952.4-012059 & 10011280269 & $23: 49: 52.4$ & $-01: 20: 59.4$ & 53637.21180 & 1.76549 & 0.0390 & 325 & $\mathrm{D}$ & $\cdots$ & $\cdots$ \\
\hline CSS_J234939.6-004257 & 10011280381 & $23: 49: 39.6$ & $-00: 42: 57.1$ & 55113.21426 & 0.49138 & 0.0345 & 324 & $\mathrm{D}$ & $\cdots$ & $\cdots$ \\
\hline CSS_J234850.3+133300 & 11121260465 & $23: 48: 50.3$ & $+13: 33: 00.1$ & 55858.22093 & 1.46550 & 0.0288 & 442 & $\mathrm{D}$ & $\ldots$ & $\ldots$ \\
\hline CSS_J234828.2+403240 & 11400990337 & $23: 48: 28.2$ & $+40: 32: 40.1$ & 55119.12598 & 0.93421 & 0.0182 & 219 & $\mathrm{~N} / \mathrm{A}$ & $\ldots$ & $\ldots$ \\
\hline CSS_J234827.2+392032 & 11381030834 & $23: 48: 27.2$ & $+39: 20: 32.8$ & 55348.41153 & 1.70451 & 0.0172 & 308 & $\mathrm{~N} / \mathrm{A}$ & $\cdots$ & $\cdots$ \\
\hline CSS_J234826.5+271203 & 11261160472 & $23: 48: 26.5$ & $+27: 12: 03.6$ & 54732.19943 & 0.86780 & 0.0237 & 371 & $\mathrm{D}$ & $\cdots$ & $\ldots$ \\
\hline CSS_J234819.9+344833 & 11351060279 & $23: 48: 19.9$ & $+34: 48: 33.9$ & 55943.75582 & 2.52099 & 0.0130 & 326 & $\mathrm{~N} / \mathrm{A}$ & $\cdots$ & $\cdots$ \\
\hline CSS_J234734.4+203331 & 11211200175 & $23: 47: 34.4$ & $+20: 33: 31.9$ & 55088.41305 & 0.86457 & 0.0144 & 429 & $\mathrm{D}$ & $\cdots$ & $\cdots$ \\
\hline CSS_J234700.0+180015 & 11181220236 & $23: 47: 00.0$ & $+18: 00: 15.6$ & 54480.10645 & 3.07628 & 0.0169 & 431 & $\mathrm{D}$ & $\cdots$ & $\cdots$ \\
\hline CSS_J234554.3-003131 & 10011270462 & $23: 45: 54.3$ & $-00: 31: 31.1$ & 54477.07608 & 0.69255 & 0.0234 & 398 & $\mathrm{D}$ & $\cdots$ & $\ldots$ \\
\hline CSS_J234502.5+415419 & 11400980842 & $23: 45: 02.5$ & $+41: 54: 19.9$ & 54632.41237 & 0.85779 & 0.0763 & 245 & $\mathrm{D}$ & $\cdots$ & $\cdots$ \\
\hline CSS_J234439.7+055255 & 11071260052 & $23: 44: 39.7$ & $+05: 52: 55.8$ & 56301.08055 & 0.50740 & 0.0136 & 407 & SD & $\cdots$ & $\cdots$ \\
\hline CSS_J234348.2+270630 & 11261150467 & $23: 43: 48.2$ & $+27: 06: 30.4$ & 55024.49074 & 2.15783 & 0.0135 & 438 & $\mathrm{D}$ & $\ldots$ & $\ldots$ \\
\hline CSS_J234339.1+362901 & 11351050807 & $23: 43: 39.1$ & $+36: 29: 01.2$ & 55366.42500 & 0.31764 & 0.1027 & 386 & $\mathrm{D}$ & $\cdots$ & $\cdots$ \\
\hline CSS_J234331.2-010354 & 10011270364 & $23: 43: 31.2$ & $-01: 03: 54.4$ & 54009.17422 & 1.13869 & 0.0147 & 398 & $\mathrm{D}$ & $\cdots$ & $\cdots$ \\
\hline CSS_J234306.1+060347 & 11071260081 & $23: 43: 06.1$ & $+06: 03: 47.7$ & 54730.34298 & 3.6267 & 0.0335 & 407 & $\mathrm{D}$ & $\cdots$ & $\cdots$ \\
\hline CSS_J234230.6+410139 & 11400980526 & $23: 42: 30.6$ & $+41: 01: 39.8$ & 54394.14236 & 0.52837 & 0.0256 & 248 & $\mathrm{D}$ & $\cdots$ & $\cdots$ \\
\hline CSS_J234137.0+264453 & 11261150363 & $23: 41: 37.0$ & $+26: 44: 53.2$ & 55009.56074 & 0.33624 & 0.1115 & 436 & $\mathrm{D}$ & $\cdots$ & $\cdots$ \\
\hline CSS_J234116.3+392234 & 11381020853 & $23: 41: 16.3$ & $+39: 22: 34.6$ & 53655.20953 & 0.70380 & 0.0213 & 325 & $\mathrm{D}$ & $\cdots$ & $\cdots$ \\
\hline
\end{tabular}

Notes.

${ }^{\text {a }}$ Epoch at primary minimum.

${ }^{\mathrm{b}}$ Mean photometric error $\left(V_{C S S}\right)$.

(This table is available in its entirety in machine-readable form.)

maximum light out of the eclipses (MaxI), the difference between the eclipse depths (MinI-MinII), the amplitude (Amp), and the mean magnitude $\left(\left\langle V_{\mathrm{mag}}\right\rangle\right)$. The histograms of the distribution of errors for the phenomenological parameters are shown in Figure 5.

\section{Classification}

The EBs in previous CSS data releases were classified into D or SD systems based on visual inspection of the LCs. Lee
(2015), using the Method for Eclipsing Component Identification (Devor \& Charbonneau 2006), found 272 SD EBs among 2170 fitted LCs (of the total 4683), based on Roche lobe filling criteria.

Based on the system morphology classification, we performed, for the first time, an automated classification of the majority of EA-type CSS EBs with machine-learning algorithms. In our search, unsupervised machine-learning followed, by supervised learning, was performed using 8000 synthetic LCs of D, SD, 

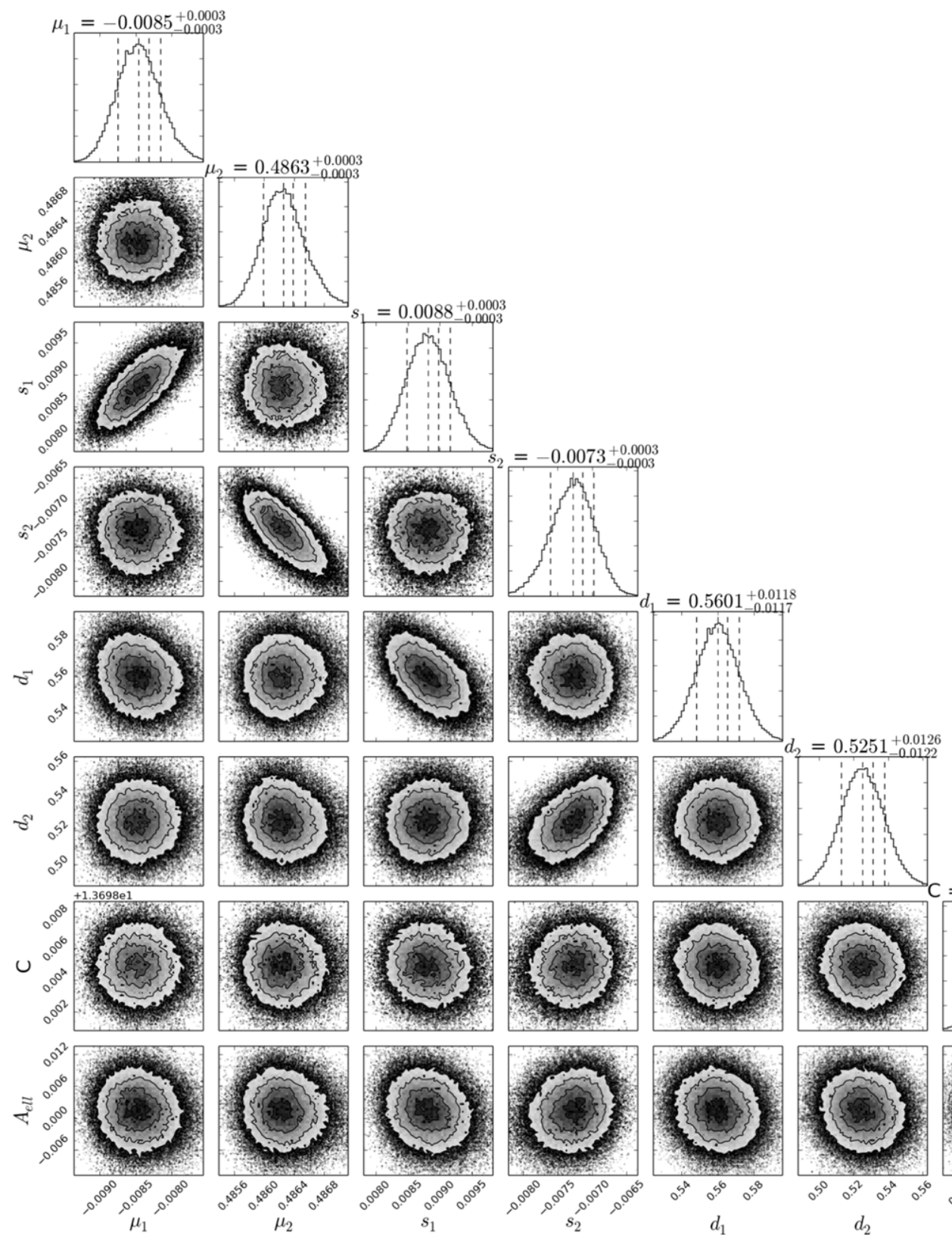
$=13.7029_{-0.0013}^{+0.0014}$
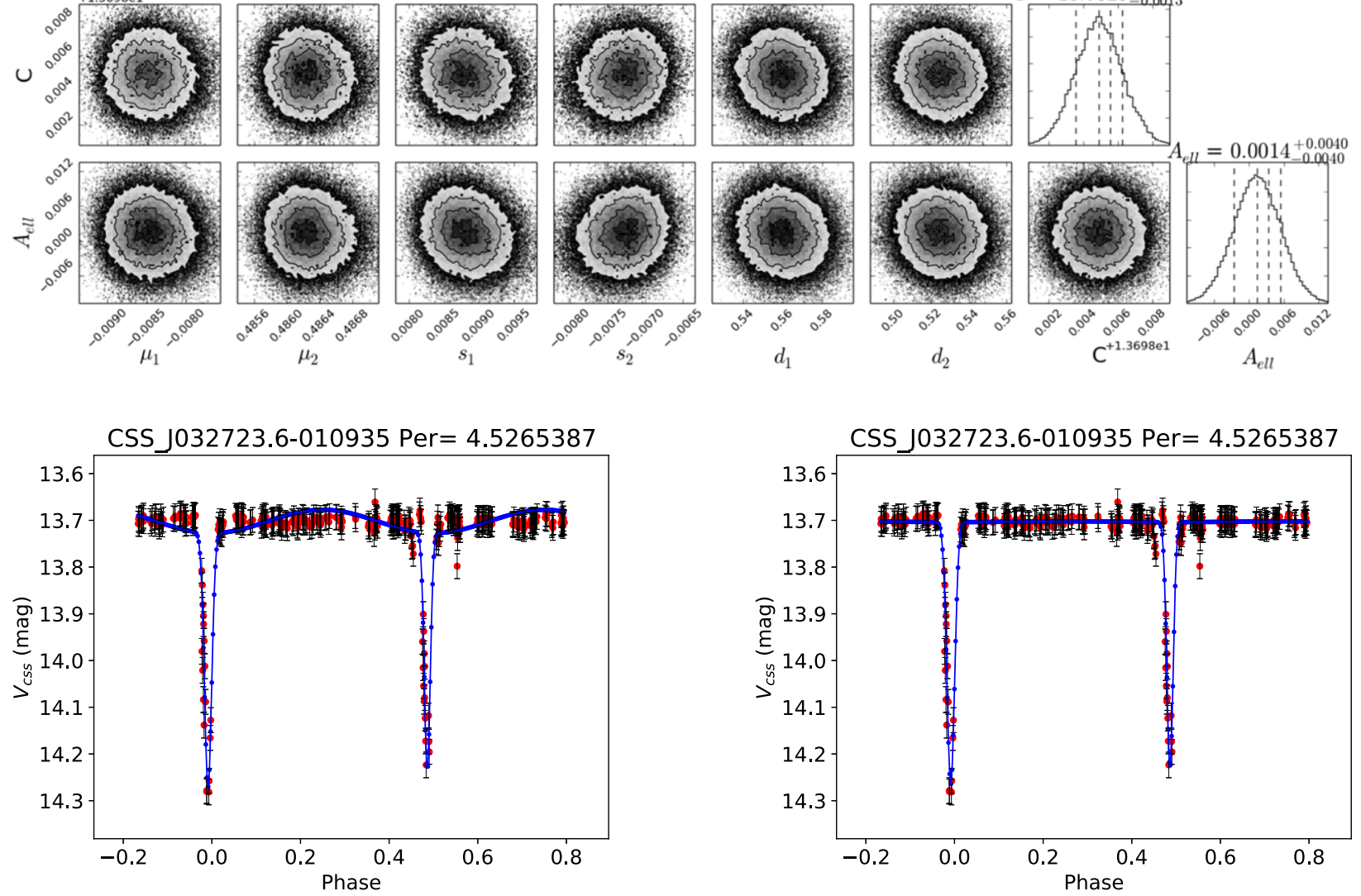

Figure 3. Top: one-dimensional and two-dimensional projections of the posterior probability distributions (Foreman-Mackey et al. 2014) of a few parameters inferred from the TGM on each light curve. Bottom: example light curve with the initial (left) and the final (right) TGM fitting coupled by MCMC. The blue dots and solid lines refer to the resulting TGM, while the red dots refer to the CSS data. CSS IDs and periods are given at top of each light curve. 

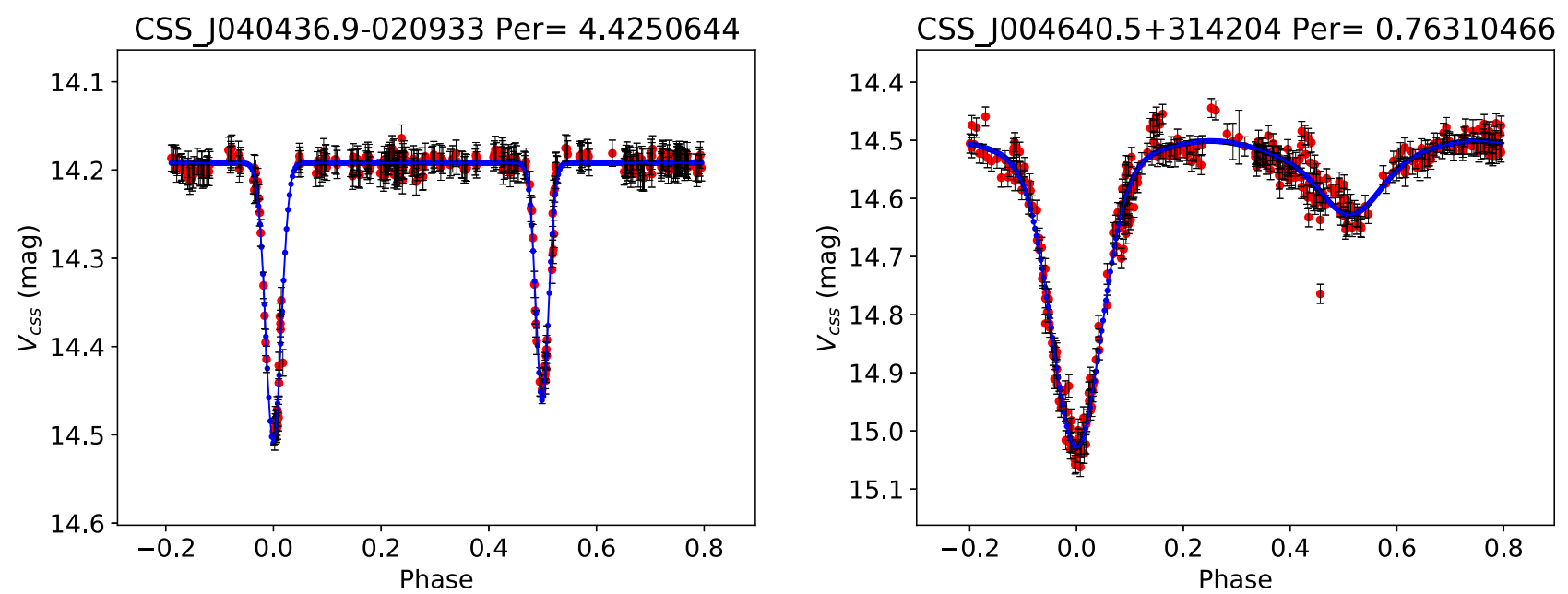

Figure 4. Representative examples of a D system (left) and an SD system (right), obtained using TGM fitting. The symbols and colors are the same as those in Figure 3. CSS IDs and periods are given on top of each light curve.

Table 2

Phenomenological Parameters of 4680 EBs

\begin{tabular}{|c|c|c|c|c|c|c|c|c|c|c|}
\hline Name & $\begin{array}{l}\text { Amp } \\
\text { (mag) }\end{array}$ & $\begin{array}{c}\mathrm{Amp}_{\mathrm{err}}^{\mathrm{a}} \\
(\mathrm{mag})\end{array}$ & $\begin{array}{l}\text { MinI } \\
(\mathrm{mag})\end{array}$ & $\begin{array}{c}\operatorname{MinI}_{\text {err }}^{\mathrm{a}} \\
(\mathrm{mag})\end{array}$ & $\begin{array}{l}\text { MinII } \\
(\mathrm{mag})\end{array}$ & $\begin{array}{c}\operatorname{MinII}_{\text {err }}{ }^{a} \\
(\mathrm{mag})\end{array}$ & $\begin{array}{l}\text { MaxI } \\
(\mathrm{mag})\end{array}$ & $\begin{array}{c}\operatorname{MaxI}_{\text {err }}{ }^{a} \\
(\mathrm{mag})\end{array}$ & $\underset{(\mathrm{mag})}{|\operatorname{MinI}-M i n I I|}$ & $\underset{(\mathrm{mag})}{\mid \mathrm{MinI}^{2}}$ \\
\hline CSS_J235945.5+303731 & 1.9357 & 0.0225 & 15.6800 & 0.0205 & 13.8708 & 0.0130 & 13.7176 & 0.0145 & 1.8092 & 0.0216 \\
\hline CSS_J235856.7+371823 & 0.2647 & 0.0083 & 13.5267 & 0.0061 & 13.3597 & 0.0069 & 13.2358 & 0.0062 & 0.1669 & 0.0088 \\
\hline CSS J235816.7+293325 & 1.4841 & 0.0276 & 18.2052 & 0.0231 & 16.9536 & 0.0242 & 16.6789 & 0.0180 & 1.2516 & 0.0321 \\
\hline CSS_J235756.9-023247 & 0.6316 & 0.0344 & 13.3882 & 0.0269 & 13.2883 & 0.0288 & 12.7309 & 0.0239 & 0.0999 & 0.0379 \\
\hline CSS_J235715.5+305455 & 0.3071 & 0.0087 & 14.6847 & 0.0082 & 14.6709 & 0.0076 & 14.3497 & 0.0031 & 0.0137 & 0.0111 \\
\hline CSS_J235538.3+384723 & 0.2798 & 0.0150 & 16.6196 & 0.0117 & 16.4139 & 0.0152 & 16.3397 & 0.0104 & 0.2057 & 0.0192 \\
\hline CSS_J235444.8+305751 & 0.8093 & 0.0271 & 16.7195 & 0.0188 & 16.5878 & 0.0268 & 15.8768 & 0.0214 & 0.1317 & 0.0316 \\
\hline CSS_J235401.4+374029 & 0.3562 & 0.0283 & 15.7998 & 0.0205 & 15.7252 & 0.0240 & 15.4119 & 0.0217 & 0.0745 & 0.0301 \\
\hline CSS_J235313.6-021850 & 0.7300 & 0.0209 & 15.0009 & 0.0173 & 14.8783 & 0.0214 & 14.2432 & 0.0132 & 0.1225 & 0.0268 \\
\hline CSS_J235227.0+395515 & 0.7084 & 0.0145 & 16.6152 & 0.0112 & 16.0845 & 0.0100 & 15.8725 & 0.0103 & 0.5306 & 0.0143 \\
\hline CSS_J235151.3+035409 & 1.0865 & 0.0186 & 14.3761 & 0.0159 & 13.3307 & 0.0121 & 13.2895 & 0.0107 & 1.0454 & 0.0200 \\
\hline CSS_J235104.0+115651 & 0.3206 & 0.0128 & 14.0039 & 0.0105 & 13.9033 & 0.0130 & 13.6566 & 0.0079 & 0.1006 & 0.0164 \\
\hline CSS_J234952.4-012059 & 1.0510 & 0.0264 & 17.5661 & 0.0219 & 16.7182 & 0.0194 & 16.4766 & 0.0165 & 0.8479 & 0.0283 \\
\hline CSS_J234939.6-004257 & 0.4143 & 0.0217 & 16.7682 & 0.0166 & 16.4422 & 0.0153 & 16.3179 & 0.0161 & 0.3259 & 0.0211 \\
\hline CSS_J234850.3+133300 & 0.5252 & 0.0190 & 16.4847 & 0.0175 & 16.2129 & 0.0172 & 15.9255 & 0.0081 & 0.2718 & 0.0243 \\
\hline CSS_J234828.2+403240 & 0.2586 & 0.0110 & 15.1143 & 0.0091 & 15.1082 & 0.0153 & 14.8268 & 0.0069 & 0.0061 & 0.0175 \\
\hline CSS_J234827.2+392032 & 0.3341 & 0.0140 & 14.9943 & 0.0120 & 14.7398 & 0.0562 & 14.6316 & 0.0079 & 0.2544 & 0.0574 \\
\hline CSS_J234826.5+271203 & 0.4481 & 0.0109 & 15.9527 & 0.0088 & 15.8867 & 0.0097 & 15.4728 & 0.0072 & 0.0659 & 0.0128 \\
\hline CSS_J234819.9+344833 & 1.3043 & 0.0420 & 12.7552 & 0.0366 & 11.8263 & 0.0086 & 11.4509 & 0.0230 & 0.9289 & 0.0376 \\
\hline CSS_J234734.4+203331 & 0.7597 & 0.0145 & 14.8161 & 0.0123 & 14.3558 & 0.0187 & 14.0290 & 0.0083 & 0.4602 & 0.0222 \\
\hline CSS_J234700.0+180015 & 0.3179 & 0.0088 & 14.9473 & 0.0072 & 14.9158 & 0.0085 & 14.6010 & 0.0056 & 0.0315 & 0.0109 \\
\hline CSS_J234554.3-003131 & 0.8222 & 0.0161 & 16.3255 & 0.0132 & 15.8831 & 0.0151 & 15.4719 & 0.0103 & 0.4424 & 0.0195 \\
\hline CSS_J234502.5+415419 & 0.6371 & 0.0464 & 18.1580 & 0.0394 & 18.0035 & 0.0285 & 17.4683 & 0.0268 & 0.1544 & 0.0473 \\
\hline CSS_J234439.7+055255 & 0.4433 & 0.0165 & 13.5755 & 0.0130 & 13.3442 & 0.0142 & 13.1060 & 0.0112 & 0.2313 & 0.0187 \\
\hline CSS_J234348.2+270630 & 0.5531 & 0.0119 & 13.7762 & 0.0108 & 13.7734 & 0.0200 & 13.1970 & 0.0054 & 0.0028 & 0.0226 \\
\hline CSS_J234339.1+362901 & 0.7290 & 0.0426 & 18.6783 & 0.0311 & 18.3661 & 0.0364 & 17.8895 & 0.0323 & 0.3121 & 0.0458 \\
\hline CSS_J234331.2-010354 & 0.1887 & 0.0051 & 14.3634 & 0.0045 & 14.2967 & 0.0074 & 14.1473 & 0.0025 & 0.0667 & 0.0086 \\
\hline CSS_J234306.1+060347 & 0.8140 & 0.0196 & 17.0581 & 0.0188 & 16.6652 & 0.0158 & 16.2079 & 0.0060 & 0.3928 & 0.0244 \\
\hline CSS_J234230.6+410139 & 0.3710 & 0.0140 & 16.0309 & 0.0107 & 15.7939 & 0.0117 & 15.6270 & 0.0100 & 0.2369 & 0.0153 \\
\hline CSS_J234137.0+264453 & 0.7832 & 0.0390 & 18.7695 & 0.0323 & 18.4569 & 0.0328 & 17.9221 & 0.0261 & 0.3125 & 0.0438 \\
\hline CSS_J234116.3+392234 & 0.6616 & 0.0150 & 15.8778 & 0.0119 & 15.3690 & 0.0106 & 15.1853 & 0.0101 & 0.5088 & 0.0154 \\
\hline
\end{tabular}

Note.

${ }^{\mathrm{a}}$ Estimated from the fitting.

(This table is available in its entirety in machine-readable form.)

overcontact (OC), and ellipsoidal (ELL) EBs. As a training set, 2000 LCs were randomly selected for each class, out of a total of $\sim 32,000$ synthetic LCs. The synthetic LCs were created by a Monte Carlo-based script (Prša et al. 2008) in PHOEBE-scripter (Prša \& Zwitter 2005), using randomly selected parameters for each physical model. For each LC, 201 equally phased bins in the range $[0,1]$ were utilized.

An unsupervised learning was then performed for each of 4050 phenomenological models obtained from Section 3.2, selected according to fitting performance. This was done by 


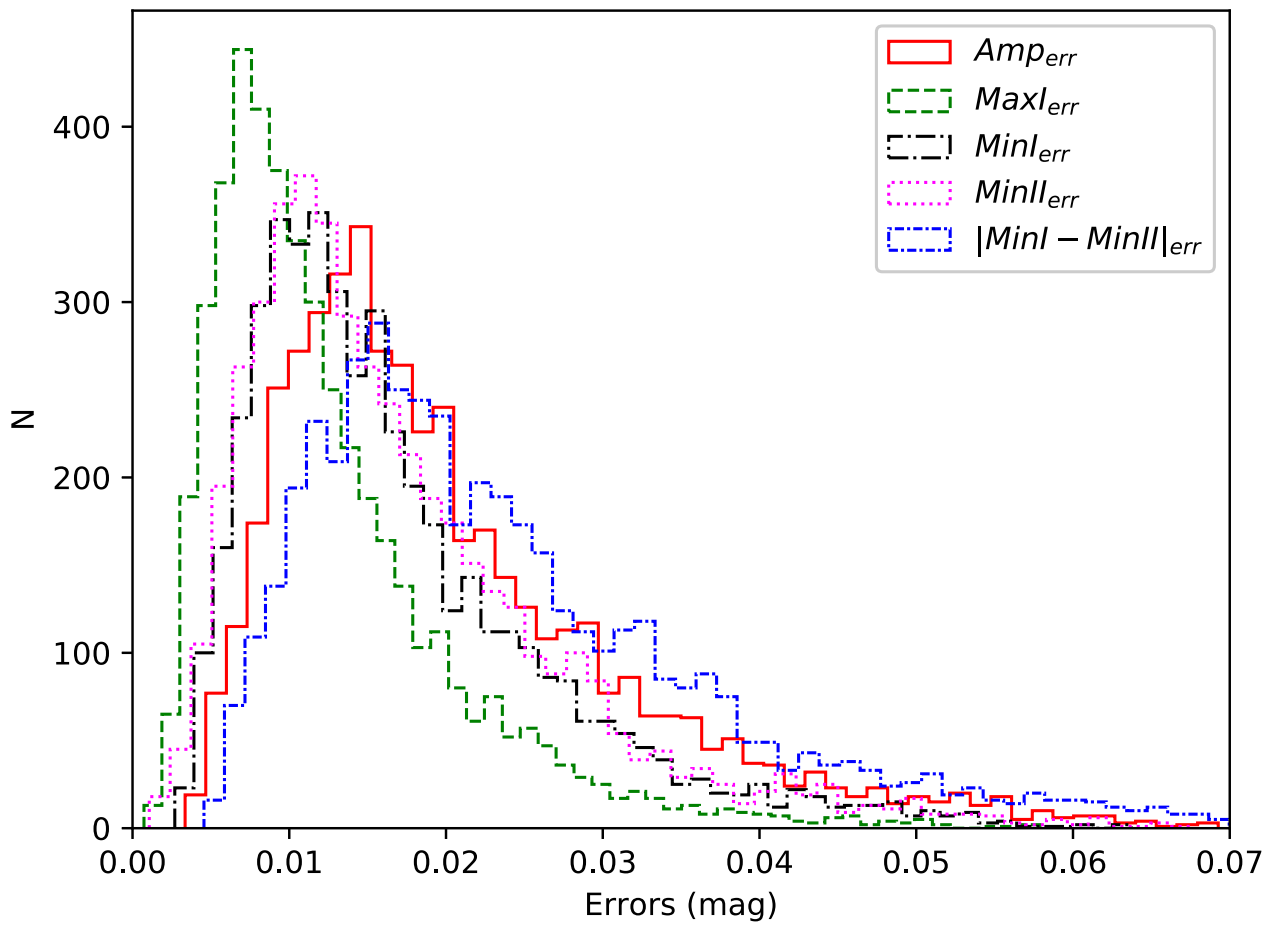

Figure 5. Distribution of the obtained uncertainties in the parameters Amp, MaxI, MinI, MinII, and the difference |MinI-MinII|.

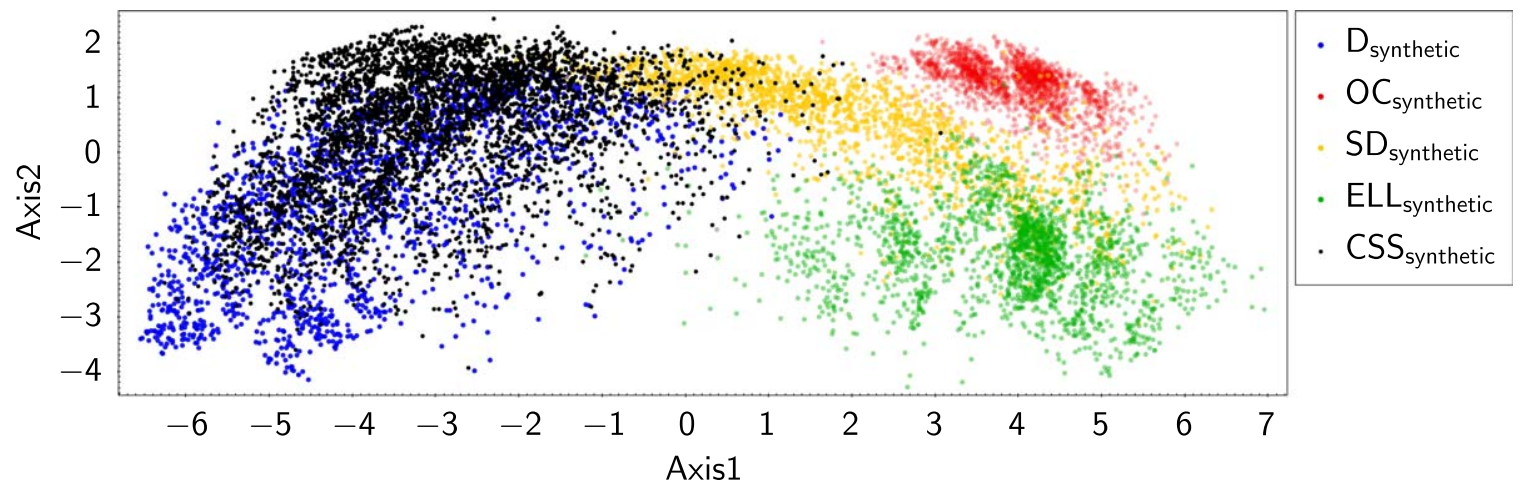

Figure 6. Lower-dimensional input data space projection (2D projection) applying the method of Isomap. The axes of the 2D projection represent the top two eigenvectors of the geodesic distance matrix. The colored symbols indicate the distribution of synthetic LCs, whereas the black dots indicate the positions of CSS sources.

applying a variety of methods through the scikit-learn ${ }^{12}$ module (Pedregosa et al. 2012) in Python. Lower-dimensional space projections (2D and 3D) were found by applying the method of complete isometric feature mapping with 160 nearest neighbors (Isomap, Tenenbaum et al. 2000) that separated the classes (Figure 6). Considering the separation of the classes, we applied a supervised machine-learning using the values of the 3D projection as input for the training set and the CSS data.

Furthermore, random Gaussian noise with a $\sigma=0.08 \mathrm{mag}$ was added to the sample of the training set and the phenomenological models. A variety of classifiers were applied, and we found that the best performance (validation score 92\%) was achieved by Support Vector Machine (SVM). However, similar validation scores $(\sim 89 \%-91 \%)$ were achieved using Random Forest, Artificial Neural Network (ANN), and $\mathrm{K}$-nearest neighbor (KN) classifiers.

\footnotetext{
$\overline{12 \text { http://scikit-learn.org/stable/index.html }}$
}

Table 3

Confusion Matrix of the SVM Classifier on 2000 Synthetic Test EBs

\begin{tabular}{lcccc}
\hline \hline & $\mathrm{D}$ & $\mathrm{SD}$ & $\mathrm{OC}$ & ELL \\
\hline $\mathrm{D}$ & 525 & 1 & 0 & 1 \\
$\mathrm{SD}$ & 3 & 411 & 8 & 77 \\
$\mathrm{OC}$ & 0 & 4 & 470 & 2 \\
ELL & 1 & 22 & 4 & 471 \\
contam. & 0.01 & 0.06 & 0.02 & 0.14 \\
\hline
\end{tabular}

The confusion matrix of 2000 test synthetic EBs from the SVM classifier is presented in Table 3 . We found a discrepancy among the classifiers for 263 EBs. Finally, after visual inspection, 54 systems were classified into D, 64 into SD, and 145 into $\mathrm{D} / \mathrm{SD}$. Therefore, the final catalog, presented in Table 1, contains $3456 \mathrm{D}(85 \%), 449 \mathrm{SD}(11 \%)$, and $145 \mathrm{EBs}$ $(4 \%)$ with uncertain classification $(\mathrm{D} / \mathrm{SD})$. 

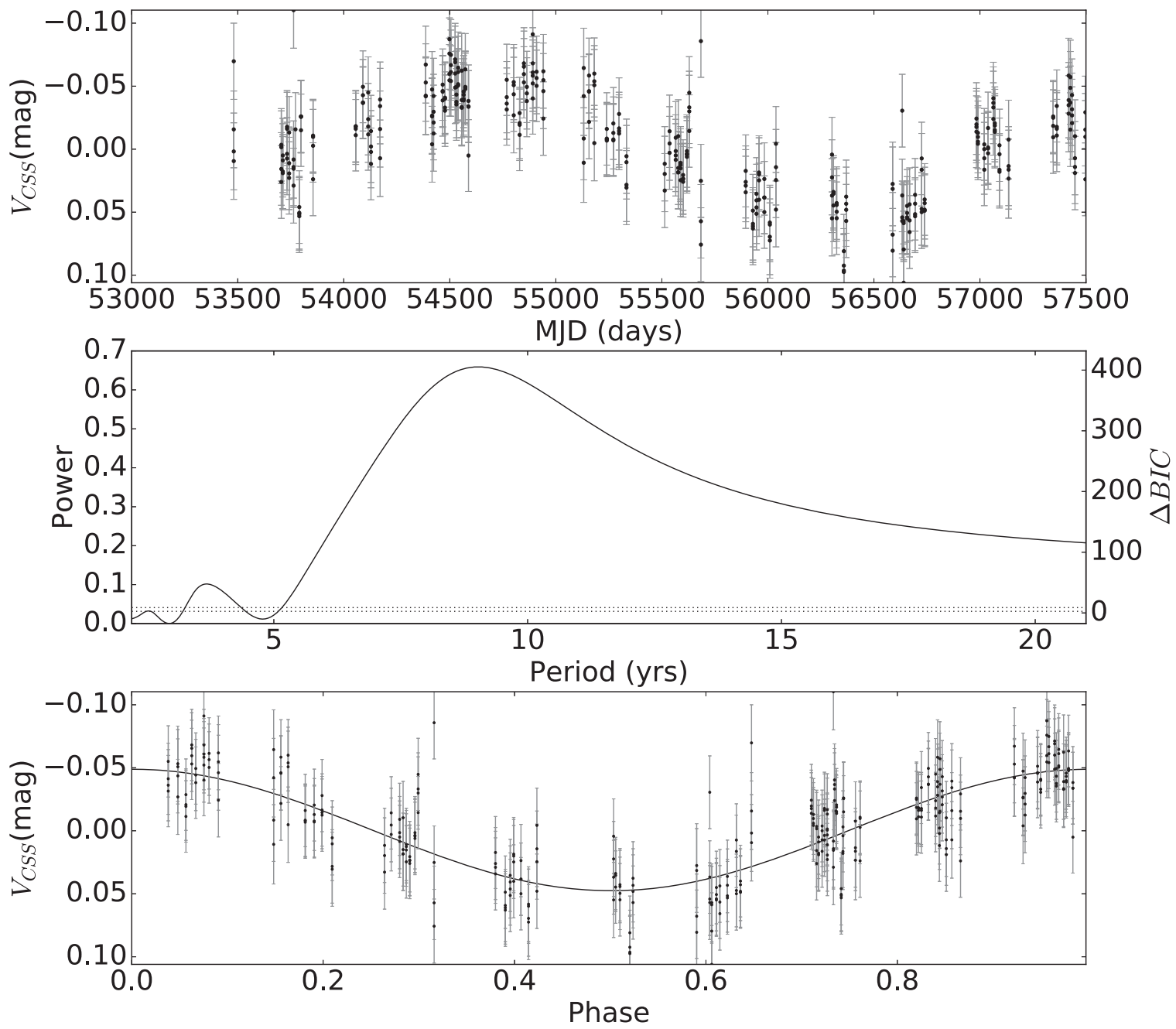

Figure 7. Representative example of an EB (CSS J083938.7-050614) showing long-term variation in maximum light. Shown here is the result from applying a GLS periodogram to the residuals after subtracting the phenomenological model. Top: the residuals of the time-series data after subtraction of the TGM phenomenological model. Middle: GLS periodogram analysis of the residuals as a function of time. The dashed lines represent the $1 \sigma$ and $3 \sigma$ significance levels derived from 1000 Monte Carlo re-samplings. Bottom: residuals of the time-series data phased with the period derived from the periodogram.

\section{Long-term Variations}

Many phased LCs revealed scattering around maximum light, i.e., different maxima in brightness, as shown in Figures 7 and 8 . This made us search for possible long-term changes over the 12-year time span of observations.

To detect such variations, we applied three methods. In the first one (Method 1) we subtracted the TGM phenomenological model from the time-series observations (Figure 7, top) and performed a GLS analysis of the residuals (Figure 7, middle), in order to evaluate the possible presence of periodicity in this variation (Figure 7, bottom). For the second and third methods, prior to the fitting, the LCs were binned in time, with bin sizes that depended on the dynamical range of observations, and the median value and standard deviation were calculated for each such bin. Then, the eclipses were removed by selecting the data points in the neighborhood of the median values, applying $1 \sigma$ tolerance (Figure 8, top right). The amplitude and the period of binned LCs were calculated through a GLS periodogram using the FATS library (Nun et al. 2015) in Python (Method 2) or by applying a harmonic fit to the binned data (Method 3). In order to detect significant variations over long ( $\sim 5-10$ years) timescales, the following set of constrains was applied to the results of the above methods:

1. LCs with amplitudes of the maxima variation lower than the LC mean error were rejected.

2. LCs with periods of maxima variation $\lesssim 800$ days or $\gtrsim 7000$ days were rejected, due to the available time span of the observations. The upper limit is set by the fact that the total time baseline of the current sample of data of CSS survey is about 12 years. Thus, the period of any parabolic variation must be less than roughly $1.5 \times 12$ years.

3. Only signals with a Bayesian Information Criterion (BIC, Schwarz 1978) greater than 15 were accepted.

4. Peak GLS power must be five times the $3 \sigma$ power as predicted by 1000 Monte Carlo re-samplings (VanderPlas et al. 2012, 2014; Marsh et al. 2017).

Combining the results from the previous methods and applying the aforementioned criteria, we found 152 systems in the sample of 4680 EBs that appear to exhibit variability in their maxima. For all these systems, the variability seems to be either periodic or quasi-periodic, over long ( $\sim 5-10$ years) 

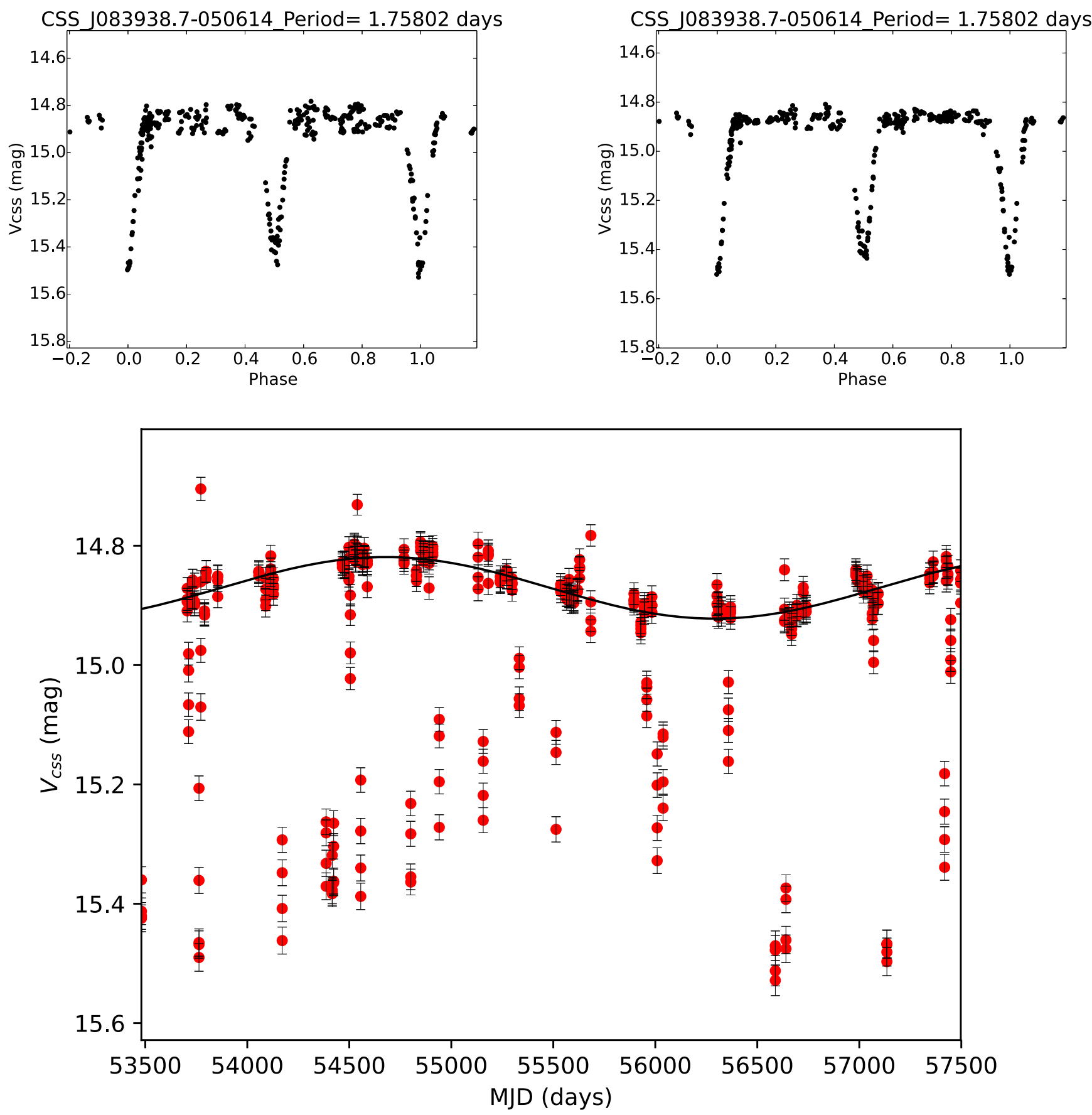

Figure 8. Top: for the same star shown in Figure 7, we show here the light curves before (left) and after (right) removal of the long-term trend. Bottom: a harmonic fitting (solid line) was performed on the maximum light after the removal of the eclipses using the method described in Section 5.

timescales. Figure 8 (bottom) shows a representative example with the derived sinusoid model (Method 3) fitted on timebinned data.

The resulting sample was examined for the possibility of the presence of SDSS (Ahn et al. 2012; Alam et al. 2015) sources within $5^{\prime \prime}$ of our systems, as any such nearby sources could contaminate the CSS photometry and thus produce spurious variations in the LCs. As a result, 33 EBs were removed from the sample, resulting in 119 EBs with maximum light variation. This final sample of 119 EBs showing long-term variations in the maximum light is labeled in Table 1 as "LongTerm +."

\subsection{Applegate Mechanism versus Spot Activity}

Maximum light variations in EBs can be explained by the Applegate mechanism (Applegate 1992) that relates the orbital period modulation to the operation of a hydromagnetic dynamo in the convection zone of the active star in a close binary system. As the active star progresses through its magnetic activity cycle, a changing differential rotation modifies its shape, changing the gravitational quadrupole moment that manifests itself through a cyclically varying orbital period and luminosity of the star, with the same period as the magnetic cycle. Fractional luminosity variations of $\Delta L / L \sim 0.1$ of the 

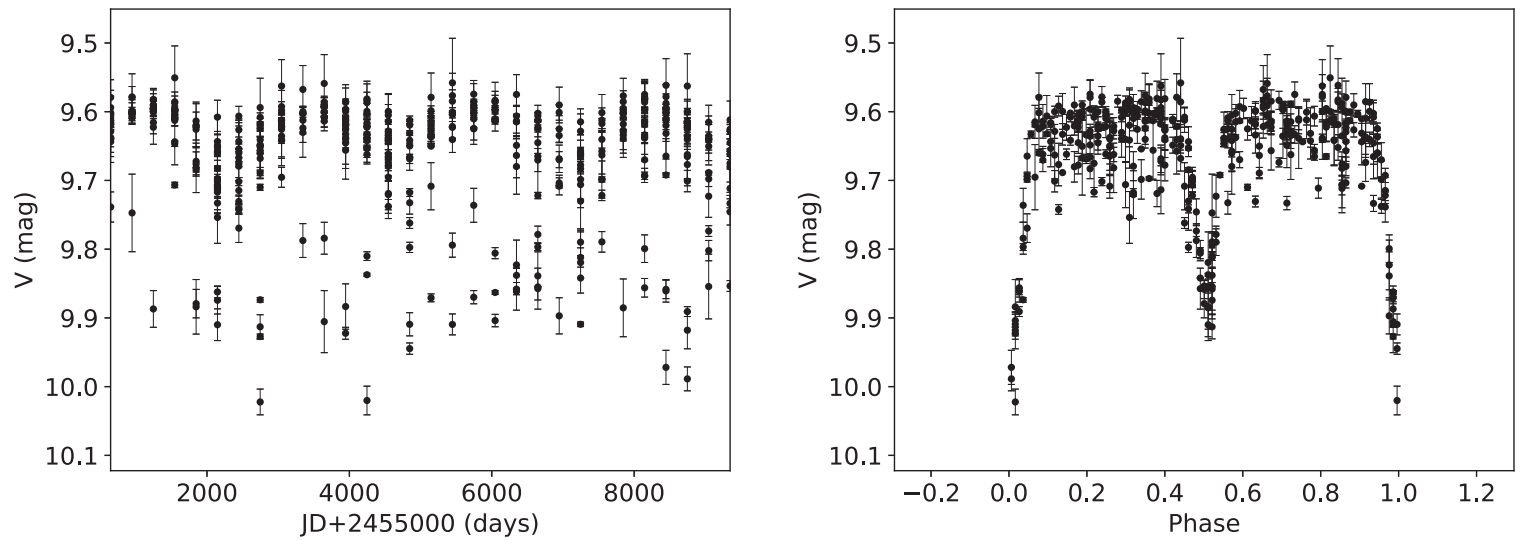

Figure 9. Time-series (left) and phase-folded data (right) of a simulated spotted EB with a magnetic cycle of 6.3 years.

active(s) star(s) can produce period variations of $\Delta P / P \sim 10^{-5}$. The majority of our 119 candidates matching Two Micron All-Sky Survey (2MASS; Skrutskie et al. 2006) sources have colors $J-H>0.237 \mathrm{mag}$ and $H-K>0.063$ mag, which implies effective temperatures likely lower than $\sim 6200 \mathrm{~K}$ (Pecaut \& Mamajek 2013). Since our results are in agreement with the changes expected under the Applegate mechanism, the latter cannot be excluded as an explanation of the detected maximum light variations. However, if the convective zone cannot respond fast enough (i.e., the thermal timescale of the envelope is much longer than the timescale of the activity cycles), the heat flow variations will be dumped, and thus become unobservable (Watson \& Marsh 2010; Khaliullin \& Khaliullina 2012).

On the other hand, maximum light variations could be explained by cool starspot coverage due to the magnetic activity. Our Sun shows such variations in a cycle of 11 years, and in case of low-mass EBs where the components are rotating nearly 100 times faster than the Sun, the deep convective envelope, along with rapid rotation, can produce a strong magnetic dynamo and solar-like magnetic activity. Long LCs shed light on the nature of the stellar activity of solar or late-type stars, either as single or as members of binary systems (such as BY Dra and RS CVn stars; Lehtinen et al. 2016). Marsh et al. (2017) suggested that the variation of the overall brightness in W UMa-type stars is probably caused by groups of starspots, rather than individual starspots.

In order to simulate the effect of maximum light variations due to starspots, a synthetic eclipsing binary was constructed using the PHOEBE-2.0 engine (Prša et al. 2016) with two main-sequence stars with effective temperatures 5880 and $5490 \mathrm{~K}$. The inclination of the system was set to $80^{\circ}$. We assumed four starspot regions in order to simulate a uniform starspot coverage. The positions (longitude and colatitude) of the starspot regions were randomly drawn from a uniform distribution, and the temperature ratio of the spot over the star was set to 0.9 for the primary (active) star. In order to simulate the magnetic cycle, we assumed cyclic variations of the starspot radius (large enough to mimic starspot regions close to maximum activity, and small for lower magnetic activity) in the range of $[0,35] \mathrm{deg}$. A period of 6.3 years was assumed for the magnetic cycle. For the virtual observations, a cadence of 300 days and a total time-span of 9000 days were assumed (i.e., we assumed that the virtual observations were carried out in one night every 300 days). Furthermore, variable random noise was added to the time-series data, and finally 300 random points were selected for the final simulated LC. The results of the simulation, under our assumptions (variable size of cool starspot regions), support the explanation of starspot activity (Figure 9). However, in order to achieve amplitudes of variation comparable to our observations and to cover regions that could produce variations also in out-of-eclipse phases, the starspot regions must be large; otherwise, we have to assume that both components show magnetic activity.

Both the Applegate mechanism and starspot activity share the same period of the magnetic cycle of the magnetically active star. Accurate times of minimum light observations and period variation analysis are needed to investigate which mechanism(s) may be the underlying cause of these variations.

\section{Low-mass Eclipsing Binaries in the Catalina Sky Survey}

Low-mass EB systems are interesting for determining the fundamental parameters of low-mass stars, which are the most common type of star in the universe. However, recent studies have shown that they represent significant challenges to the theoretical stellar models, due to their inflated sizes, magnetic activity, and also the poorly understood way they evolve in close binary systems (Chabrier et al. 2007; Feiden 2015; Zhou et al. 2015). Our list of 4050 classified EBs enables us to search for low-mass EB candidates by imposing color criteria. Accordingly, here we apply the following cuts: $V-K_{s}>3.0$, as suggested by Hartman et al. (2011) $0.35<J-H<0.8 \mathrm{mag}$, and $H-K_{s} \leqslant 0.45 \mathrm{mag}$, based on Lépine \& Gaidos (2011) and Zhong et al. (2015).

For the color selection, we again use the 2MASS $J H K_{s}$ photometry, performing a cross-match to the 2MASS catalog (Cutri et al. 2003) within $3^{\prime \prime}$ of the positions of our stars. Where available, photometry from the APASS survey (Henden et al. 2016) was also used, to obtain the $(B-V)$ color index in order to transform the $V_{\mathrm{CSS}}$ magnitudes to Jonhson $V$. This was accomplished using the same transformation formula as presented in Drake et al. (2013):

$$
V=V_{\mathrm{CSS}}+0.31 \times(B-V)^{2}+0.04 \text {. }
$$

This selects 2377 EBs. For the rest of the cataloged EBs for which we have no visual color information, we apply the transformation from 2MASS indices to the Johnson-Cousins system provided by Bilir et al. (2008, their Equation (16)). Interstellar extinction corrections were applied to the $(B-V)$ color index of each EB using the $E(B-V)$ values from Green et al. (2015). The $V J H K_{s}$ magnitudes were also corrected 
accordingly, using extinction models obtained from a combination of Marshall et al. (2006), Green et al. (2015), and Drimmel et al. (2003), as included in the Python package mwdust ${ }^{13}$ (Bovy et al. 2016). Combining the results with the classification from Section 4, only the systems classified as D were finally accepted, resulting in 609 candidates. For distances between 1 and $4.5 \mathrm{kpc}$, the results are independent of the reddening corrections applied.

To verify whether these are all bona fide low-mass EB candidates, we performed two tests:

1. We compare their infrared colors with the theoretically expected colors of main-sequence F5-M3 dwarfs, as reported by Pecaut \& Mamajek (2013). The results are shown in the upper panel of Figure 11. The reddening vector in this plot was calculated from the mean value of the extinction of the entire sample;

2. We compare their infrared colors with the colors of stars in the largest $\mathrm{K}$ and $\mathrm{M}$ dwarf spectroscopic sample (2612 binaries) from the Large Sky Area Multi-object Fiber Spectroscopic Telescope (LAMOST, Zhong et al. 2015). The results are shown in the lower panel of Figure 11.

The periods of the final sample are in the range of [0.2-3.5] days (Figure 12).

As we can see, the large majority of our sample does indeed fall within the K5 and M3 subtypes. These 609 binaries selected as low-mass candidates are marked in Table 1 as "LMcand +." Examination of Table 1 in Lee (2015) reveals a total of $572 \mathrm{~EB}$ systems with nominal component masses $<0.6 M_{\odot}$. However, as explained in that table's header, this includes a large number of systems with uncertain solutions, and also systems with large errors $\left(>0.2 M_{\odot}\right)$ in the mass values. For this reason, to carry out a meaningful comparison between our results and those reported by Lee (2015), we restrict ourselves to systems with masses in the range covered by our sample (i.e., with spectral types later than $\mathrm{K} 5$, or masses $<0.71 M_{\odot}$ ) and with errors $<0.1 M_{\odot}$. This leads to a total of 107 systems, including 7 EBs classified as either nondetached or uncertain by our analysis, but which Lee classifies as detached. Out of the remaining 100, 72 were matched with our low-mass sample. The remaining 28 systems fall outside the limits of our color selection criteria, thus suggesting that they may not be bona-fide low-mass EB systems. Note that four systems have been verified as double-lined M-dwarf EBs (Lee \& Lin 2017; Lee 2017); all of them are included in our sample of 609 candidates, but only two appear in Lee's (2015) catalog.

In addition, in our low-mass sample of detached EBs we found candidates near the short-period cutoff at $P \sim 0.22$ day (Rucinski 1992, 1997), as can be seen from Figure 10. Only a few such systems are currently known (Drake et al. 2014a). To our knowledge, the detached system with main-sequence components with the shortest period known (0.1926 day) is GSC 2314-0530 (=1SWASP J022050.85+332047.6), identified by Norton et al. (2007) and modeled by Dimitrov \& Kjurkchieva (2010). Nefs et al. (2012) spectroscopically confirmed a detached system with a 0.18 day period containing an $\mathrm{M}$ dwarf, but without measuring radial velocities.

\section{Conclusions}

Using CSS data covering a 12-year time span, we obtained an updated catalog of 4680 EA-type EBs, with revised period

\footnotetext{
${ }^{13}$ https://github.com/jobovy/mwdust
}

determinations, phenomenological parameters of their LCs, and system morphology classifications based on machine-learning techniques. Our study includes many low-mass EB candidates, as well as systems that show additional variation in their maxima over long ( 5-10 years) timescales. Most of the new periods are in excellent agreement with those provided in the original Catalina catalogs, but significantly revised values have been obtained for $\sim 10 \%$ of the stars. A total of 3456 EBs were classified as D, 449 as SD, and $145 \mathrm{EBs}$ had an uncertain classification. Our classification agrees with the findings of Lee (2015) for $83 \%$ of the sources. The sample classified as SD contains $\sim 9 \%$ systems with spectral types earlier than F0V, thus it seems that the majority of the systems in the sample are F-G spectral type EA systems with periods of less than a day. These systems have been characterized as short-period Algols (W CrV, Rucinski \& Lu 2000) in the scenario of Stepien (2006). At the same time, they have also been described as being in near (CN And, Van Hamme et al. 2001; AX Dra, Kim et al. 2004) or broken (CN And, Van Hamme et al. 2001; Avvakumova et al. 2013) contact. We again caution, as we did in the introduction, that a detailed physical modeling of individual EBs is needed to reveal the true system configuration.

Following our methodology of searching for K- and M-type dwarfs, we ended up with a sample of 609 low-mass EB candidates, increasing the total sample of stars at the low-mass end. Spectroscopic follow-up of these sources would be useful to help place constraints on models of low-mass stars. The majority of Lee's (2015) low-mass candidates are included in our sample, including four that have been verified as doublelined M dwarf EBs (Lee \& Lin 2017; Lee 2017). Moreover, we identified rare EA systems with periods close to the period cutoff at $P \sim 0.22$ day (Rucinski 1992, 1997).

In addition to these results, our analysis of the long-term trends in the CSS data revealed cyclic or quasi-cyclic modulation of the maximum brightness on long ( $\sim 5-10$ years) timescales for as many as 119 EA systems $(2.5 \%$ of the entire sample). The $\Delta L / L$ range is within [0.04-0.13], with a mean value $\langle\Delta L / L\rangle=0.075 \pm 0.017$, while the periods are in the range of [4.5-18] years, with a mean $P=12.1 \pm 3.3$ years.

Recently, Marsh et al. (2017) reported similar behavior in 205 eclipsing W UMa-type systems from CSS (2.2\% of the target sample), finding periods in the range 4-11 years and fractional luminosity variance $\Delta L / L \approx 0.04-0.16$. Close binaries are known to be significantly more active than wide binaries and single stars (e.g., Shkolnik et al. 2010), most likely due to their being tidally locked and high rotational velocities, resulting in high levels of magnetic activity. In late types this is predicted to inflate their radii by inhibiting convective flow and increasing starspot coverage. The observed long-term variability can be explained by either the Applegate mechanism or by variable spot regions. Oláh (2006) suggested that the magnetic field interaction has more effects on the starspot activities of the main-sequence stars than does the tidal force, because these stars have much higher surface gravities. As a consequence, the main-sequence stars often show active regions at quadrature phases. It should be noted that even though the vast majority of spotted stars cannot be easily imaged with special techniques (Doppler imaging or interferometry), our sample is useful to the future study of stellar activity cycles or other associated phenomena (e.g., flares). 


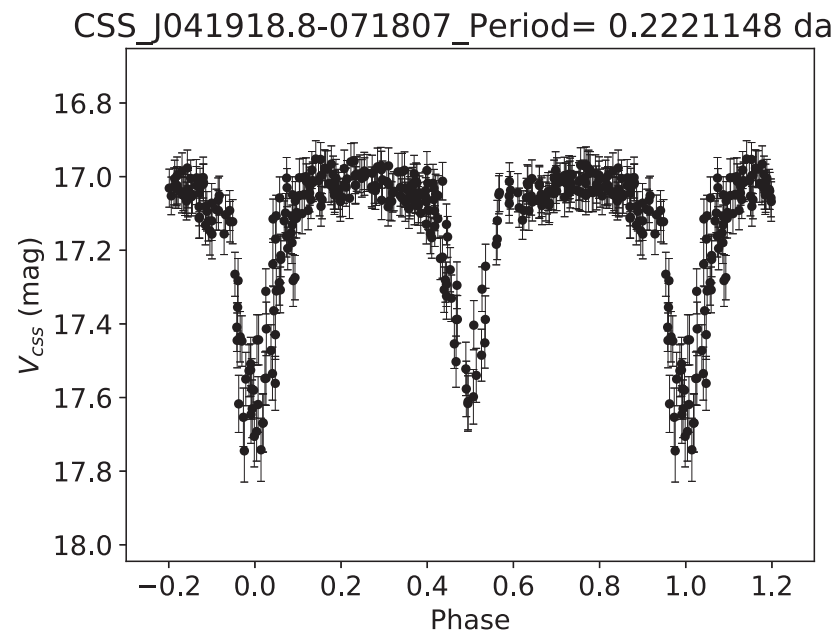

Figure 10. Folded LC of CSS J041918.8-071807, the low-mass EB candidate with the shortest period ( $P=0.22$ days) in our sample.

A.P. and M.C. gratefully acknowledge the support provided by Fondecyt through grants \#3160782 and \#1171273. Additional support for this project is provided by the Ministry for the Economy, Development, and Tourism's Millennium Science Initiative through grant IC 120009, awarded to the Millennium Institute of Astrophysics (MAS); by Proyecto Basal PFB-06/2007; and by CONICYT's PCI program through grant DPI20140066. M.C. gratefully acknowledges the additional support provided by the Carnegie Observatories through its Distinguished Scientific Visitor program. The Monte Carlo script in PHOEBE-scripter is based on the script that was kindly provided by Dr. Andrej Prša.

This work made use of data products from the CSS survey. The CSS survey is funded by the National Aeronautics and Space Administration under grant No. NNG05GF22G issued through the Science Mission Directorate Near-Earth Objects Observations Program. The CRTS survey is supported by the US National Science Foundation under grants AST-0909182, AST-1313422, AST-1413600, and AST-1518308.

This publication makes use of data products from the Two Micron All Sky Survey, which is a joint project of the University of Massachusetts and the Infrared Processing and Analysis Center/California Institute of Technology, funded by the National Aeronautics and Space Administration and the National Science Foundation. Funding for SDSS-III has been provided by the Alfred P. Sloan Foundation, the Participating Institutions, the National Science Foundation, and the U.S. Department of Energy Office of Science. The SDSS-III website is http://www.sdss3.org/.

This publication makes use of data products from SDSS-III. SDSS-III is managed by the Astrophysical Research Consortium for the Participating Institutions of the SDSS-III Collaboration including the University of Arizona, the Brazilian Participation Group, Brookhaven National Laboratory, Carnegie Mellon University, University of Florida, the French Participation Group, the German Participation Group, Harvard University, the Instituto de Astrofisica de Canarias, the Michigan State/Notre Dame/JINA Participation Group, Johns Hopkins University, Lawrence Berkeley National Laboratory, Max Planck Institute for Astrophysics, Max Planck Institute for Extraterrestrial Physics, New Mexico State University, New York University, Ohio State University, Pennsylvania State University, University of Portsmouth, Princeton University, the
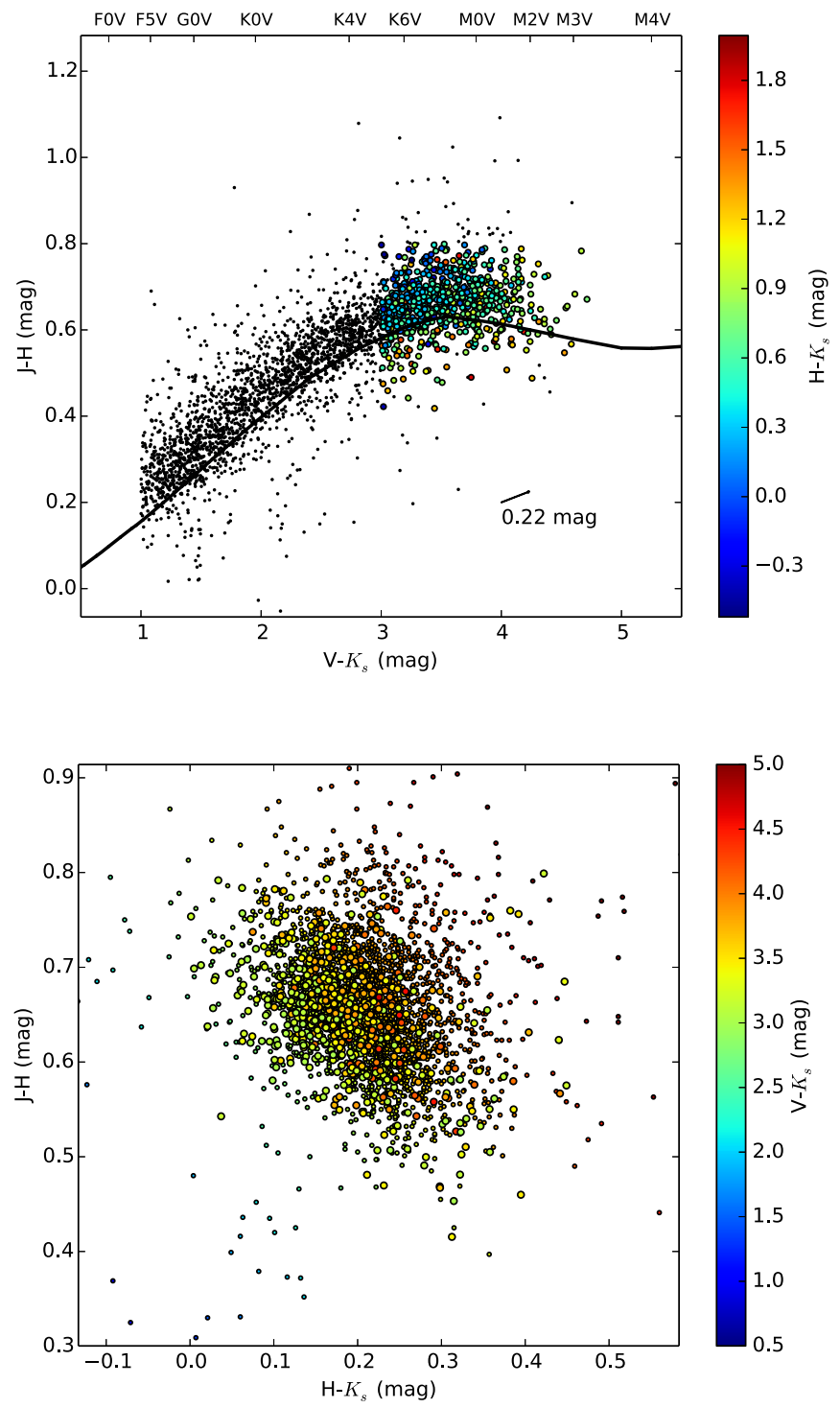

Figure 11. Top: $\left(V-K_{S}\right)-(J-H)$ color-color diagram of the 3456 CSS EBs classified as D and the theoretically expected colors of main-sequence F5M3 stars (Pecaut \& Mamajek 2013). The large dots refer to the low-mass EB candidates. The reddening vector was calculated from the mean value of the extinction of the entire sample, while the range is within [0.01-0.59] mag. Bottom: $\left(H-K_{s}\right)-(J-H)$ color-color diagram of 609 low-mass EB candidates (large dots) overplotted on the sample of low-mass stars from the LAMOST survey (smaller dots). In both panels, the different colors indicate the color index value according to the adjacent color bar.

Spanish Participation Group, University of Tokyo, University of Utah, Vanderbilt University, University of Virginia, University of Washington, and Yale University. This work has made use of the SIMBAD database, operated at CDS, Strasbourg, France.

This research was made possible through the use of the AAVSO Photometric All-Sky Survey (APASS), funded by the Robert Martin Ayers Sciences Fund.

Software: AstroML (VanderPlas et al. 2012), CKP (Protopapas et al. 2015), FATS (Nun et al. 2015), LMFIT (Newville et al. 2016), mwdust (Bovy et al. 2016), PDM (Stellingwerf 1978), PHOEBE-scripter (Prša \& Zwitter 2005), PHOEBE-2.0 (Prša et al. 2016), pyMC (Fonnesbeck et al. 2015), scikit-learn (Pedregosa et al. 2012), triangle.py-v0.1.1 (Foreman-Mackey et al. 2014), VARTOOLS (Hartman \& Bakos 2016). 


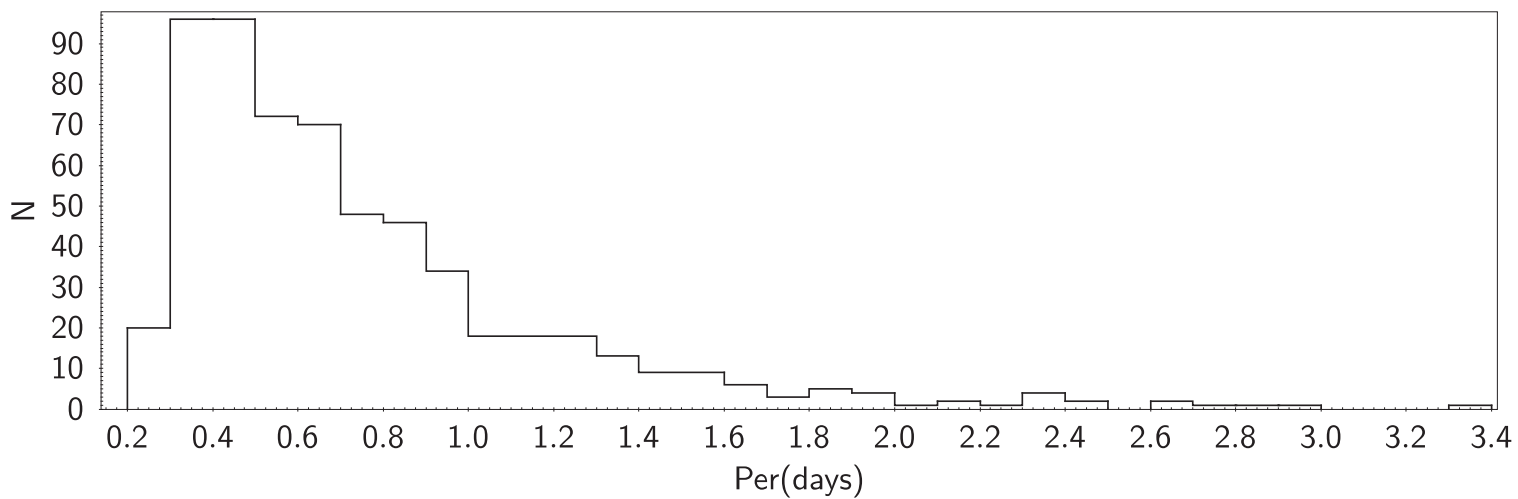

Figure 12. Period distribution of 609 low-mass EB candidates identified in this study.

\section{Appendix Acronyms and Abbreviations}

\begin{tabular}{ll}
\hline \hline & Description \\
\hline CSS & Catalina Sky Survey \\
VISTA & Visible and Infrared Survey Telescope for Astronomy \\
ASAS & All Sky Automated Survey \\
NSVS & Northern Sky Variability Survey \\
VVV & Variables in the Via Lactea \\
TrES & Transatlantic Exoplanet Survey TrES \\
OGLE & Optical Gravitational Lensing Experiment \\
HATNet & Hungarian-made Automated Telescope Network exoplanet \\
& $\quad$ survey \\
SuperWASP & Wide Angle Search for Planets \\
2MASS & Two Micron All-Sky Survey \\
APASS & AAVSO Photometric All-Sky Survey \\
LAMOST & Large Sky Area Multi-object Fiber Spectroscopic Telescope \\
CSDR2 & Catalina Surveys Data Release 2 \\
AoV & Analysis of Variance \\
BLS & Box-Least Squares \\
GLS & Generalized Lomb-Scargle \\
PDM & Phase Dispersion Minimization \\
CKP & Correntropy Kernelized Periodogram \\
MCMC & Markov Chain Monte Carlo \\
SVM & Support Vector Machine \\
ANN & Artificial Neural Network \\
KN & K-nearest neighbor \\
BIC & Bayesian Information Criterion \\
LM & Levenberg-Marquardt nonlinear minimization algorithm \\
\hline & \\
&
\end{tabular}

\section{ORCID iDs}

Athanasios Papageorgiou (1) https://orcid.org/0000-00023039-9257

Márcio Catelan (iD https://orcid.org/0000-0001-6003-8877

S. G. Djorgovski iib https://orcid.org/0000-0002-0603-3087

\section{References}

Ahn, C. P., Alexandroff, R., Allende Prieto, C., et al. 2012, ApJS, 203, 21 Alam, S., Albareti, F. D., Allende Prieto, C., et al. 2015, ApJS, 219, 12 Alonso, R., Brown, T. M., Charbonneau, D., et al. 2007, in ASP Conf. Ser. 366,

Transiting Extrapolar Planets Workshop, ed. C. Afonso, D. Weldrake, \& T. Henning (San Francisco, CA: ASP), 13

Alonso, R., Brown, T. M., Torres, G., et al. 2004, ApJL, 613, L153

Applegate, J. H. 1992, ApJ, 385, 621

Avvakumova, E. A., Malkov, O. Y., \& Kniazev, A. Y. 2013, AN, 334, 860

Bakos, G., Noyes, R. W., Kovács, G., et al. 2004, PASP, 116, 266
Bertin, E., \& Arnouts, S. 1996, A\&AS, 117, 393

Bilir, S., Ak, S., Karaali, S., et al. 2008, MNRAS, 384, 1178

Bovy, J., Rix, H.-W., Green, G. M., Schlafly, E. F., \& Finkbeiner, D. P. 2016, ApJ, 818, 130

Catelan, M., Dékány, I., Hempel, M., \& Minniti, D. 2013, BAAA, 56, 153

Catelan, M., \& Smith, H. A. 2015, Pulsating Stars (Weinheim: Wiley-VCH)

Chabrier, G., Gallardo, J., \& Baraffe, I. 2007, A\&A, 472, L17

Christian, D. J., Pollacco, D. L., Skillen, I., et al. 2006, AN, 327, 800

Cutri, R. M., Skrutskie, M. F., van Dyk, S., et al. 2003, yCat, 2246

Devor, J. 2005, ApJ, 628, 411

Devor, J., \& Charbonneau, D. 2006, ApJ, 653, 647

Dimitrov, D. P., \& Kjurkchieva, D. P. 2010, MNRAS, 406, 2559

Drake, A. J., Catelan, M., Djorgovski, S. G., et al. 2013, ApJ, 763, 32

Drake, A. J., Djorgovski, S. G., Catelan, M., et al. 2017, MNRAS, 469, 3688

Drake, A. J., Djorgovski, S. G., García-Álvarez, D., et al. 2014a, ApJ, 790, 157

Drake, A. J., Djorgovski, S. G., Mahabal, A., et al. 2009, ApJ, 696, 870

Drake, A. J., Graham, M. J., Djorgovski, S. G., et al. 2014b, ApJS, 213, 9

Drimmel, R., Cabrera-Lavers, A., \& López-Corredoira, M. 2003, A\&A, 409, 205

Feiden, G. A. 2015, in ASP Conf Ser. 496, Living Together: Planets, Host Stars and Binaries, ed. S. M. Rucinski, G. Torres, \& M. Zejda (San Francisco, CA: ASP), 137

Fonnesbeck, C., Patil, A., Huard, D., \& Salvatier, J. 2015, PyMC: Bayesian Stochastic Modelling in Python, Astrophysics Source Code Library, ascl: 1506.005

Foreman-Mackey, D., Price-Whelan, A., Ryan, G., et al. 2014, Zenodo Software Release, 2014

Graham, M. J., Djorgovski, S. G., Drake, A. J., et al. 2017, MNRAS, 470, 4112

Green, G. M., Schlafly, E. F., Finkbeiner, D. P., et al. 2015, ApJ, 810, 25

Hartman, J. D., \& Bakos, G. Á 2016, A\&C, 17, 1

Hartman, J. D., Bakos, G. Á, Noyes, R. W., et al. 2011, AJ, 141, 166

Hastings, W. K. 1970, Biometrika, 57, 97

Henden, A. A., Levine, S., Terrell, D., et al. 2015, American Astronomical Society Meeting Abstracts \#225, 336.16.

Khaliullin, K. F., \& Khaliullina, A. I. 2012, MNRAS, 419, 3393

Kim, H.-I., Lee, J. W., Kim, C.-H., et al. 2004, PASP, 116, 931

Kovacs, G. 2017, EPJWC, 152, 01005

Kovács, G., Zucker, S., \& Mazeh, T. 2002, A\&A, 391, 369

Larson, S., Beshore, E., Hill, R., et al. 2003, BAAS, 35, 36.04

Layden, A. C. 1998 , AJ, 115, 193

Lee, C.-H. 2015, MNRAS, 454, 2946

Lee, C.-H. 2017, AJ, 153, 118

Lee, C.-H., \& Lin, C.-C. 2017, RAA, 17, 15

Lehtinen, J., Jetsu, L., Hackman, T., Kajatkari, P., \& Henry, G. W. 2016, A\&A, 588, A38

Lépine, S., \& Gaidos, E. 2011, AJ, 142, 138

Levenberg, K. 1944, QApMa, 2, 164

Marquardt, D. W. 1963, SJAM, 11, 431

Marsh, F. M., Prince, T. A., Mahabal, A. A., et al. 2017, MNRAS, 465, 4678

Marshall, D. J., Robin, A. C., Reylé, C., Schultheis, M., \& Picaud, S. 2006, A\&A, 453, 635

Minniti, D., Lucas, P. W., Emerson, J. P., et al. 2010, NewA, 15, 433

Mowlavi, N., Lecoeur-Taïbi, I., Holl, B., et al. 2017, A\&A, 606, A92

Nefs, S. V., Birkby, J. L., Snellen, I. A. G., et al. 2012, MNRAS, 425, 950 
Newville, M., Stensitzki, T., Allen, D. B., et al. 2016, Lmfit: Non-Linear LeastSquare Minimization and Curve-Fitting for Python, Astrophysics Source Code Library, ascl:1606.014

Norton, A. J., Wheatley, P. J., West, R. G., et al. 2007, A\&A, 467, 785

Nun, I., Protopapas, P., Sim, B., et al. 2015, arXiv:1506.00010

Oláh, K. 2006, Ap\&SS, 304, 145

Palaversa, L., Ivezić, Ž, Eyer, L., et al. 2013, AJ, 146, 101

Papageorgiou, A., Kleftogiannis, G., \& Christopoulou, P.-E. 2014, CoSka, 43, 470

Pecaut, M. J., \& Mamajek, E. E. 2013, ApJS, 208, 9

Pedregosa, F., Varoquaux, G., Gramfort, A., et al. 2012, arXiv:1201.0490

Pojmanski, G. 1997, AcA, 47, 467

Pojmanski, G., Pilecki, B., \& Szczygiel, D. 2005, AcA, 55, 275

Pollacco, D., Skillen, I., Collier Cameron, A., et al. 2006, Ap\&SS, 304, 253

Press, W. H., Teukolsky, S. A., Vetterling, W. T., \& Flannery, B. P. 1992(2nd ed.; Cambridge: Cambridge Univ. Press)

Protopapas, P., Huijse, P., Estévez, P. A., et al. 2015, ApJS, 216, 25

Prša, A., Conroy, K. E., Horvat, M., et al. 2016, ApJS, 227, 29

Prša, A., Guinan, E. F., Devinney, E. J., et al. 2008, ApJ, 687, 542

Prša, A., \& Zwitter, T. 2005, ApJ, 628, 426

Rucinski, S. M. 1992, AJ, 103, 960

Rucinski, S. M. 1997, MNRAS, 382, 393

Rucinski, S. M., \& Lu, W. 2000, MNRAS, 315, 587

Schwarz, G. 1978, AnSta, 6, 461
Schwarzenberg-Czerny, A. 1989, MNRAS, 241, 153

Shkolnik, E. L., Hebb, L., Liu, M. C., Reid, I. N., \& Collier Cameron, A. 2010, ApJ, 716, 1522

Skrutskie, M. F., Cutri, R. M., Stiening, R., et al. 2006, AJ, 131, 1163

Soszyński, I. 2017, EPJWC, 152

Stellingwerf, R. F. 1978, ApJ, 224, 953

Stepien, K. 2006, AcA, 56, 199

Stokes, G. H., Evans, J. B., Viggh, H. E. M., Shelly, F. C., \& Pearce, E. C. 2000, Icar, 148, 21

Tenenbaum, J. B., de Silva, V., \& Langford, J. C. 2000, Sci, 290, 2319

Torres, G., Andersen, J., \& Giménez, A. 2010, A\&ARv, 18, 67

Udalski, A., Szymanski, M., Kaluzny, J., Kubiak, M., \& Mateo, M. 1992, AcA, 42, 253

Van Hamme, W., Samec, R. G., Gothard, N. W., et al. 2001, AJ, 122, 3436

VanderPlas, J., Connolly, A. J., Ivezic, Z., \& Gray, A. 2012, arXiv:1411.5039

VanderPlas, J., Fouesneau, M., \& Taylor, J. 2014, AstroML: Machine learning and data mining in astronomy Astrophysics, Source Code Library, ascl: 1407.018

Watson, C. A., \& Marsh, T. R. 2010, MNRAS, 405, 2037

Welsh, W. F., Orosz, J. A., Aerts, C., et al. 2011, ApJS, 197, 4

Woźniak, P. R., Vestrand, W. T., Akerlof, C. W., et al. 2004, AJ, 127, 2436

Zechmeister, M., \& Kürster, M. 2009, A\&A, 496, 577

Zhong, J., Lépine, S., Hou, J., et al. 2015, AJ, 150, 42

Zhou, G., Bayliss, D., Hartman, J. D., et al. 2015, MNRAS, 451, 2263 